\title{
Using the Social Ecological Model to Explore Nutritional Risk in Community-Dwelling Older Adults
}

\author{
Christine (Chris) Mills, RD, BASc, MPH, PhD Student Aging and Health
}

Queen's University, Faculty of Health Sciences, School of Rehabilitation Therapy, Kingston, Ontario

\section{Introduction}

More than one-third of community-dwelling Canadians aged 65 and older are at nutritional risk (NR). NR is related to increased morbidity and mortality. It is critical to understand the factors associated with NR.

\section{Objectives}

To determine if the Social Ecological Model (SEM) can be used to explore NR in older adults.

\section{Methods}

Three theories of aging were applied to the study of nutritional risk in older adults: the social capital theory, the life-span theory of social support (social support theory), and the social ecological model. The databases CINAHL, Embase, Medline, and Google Scholar were used to identify articles related to nutritional risk and these theories of aging. After retrieving relevant articles, the three theories were analyzed to determine which best explains nutritional risk in community-dwelling older adults.

\section{Results}

SEM was the most appropriate theory. Not only can the social support and social capital theories be incorporated into SEM, but nutritional risk is affected by many different factors at all of the multiple levels within SEM. Numerous factors also interact across levels to influence nutritional risk.

\section{Conclusions}

SEM can be used to examine the factors associated with nutritional risk in communitydwelling older adults. Understanding the factors is critical for improving NR in older adults.

\section{Significance to Dietetics}

Dietitians are best positioned to assess older adults for NR and to implement nutrition interventions designed to address or mitigate contributing factors. SEM can be used to guide the development and evaluation of these interventions. If nutritional risk can be reduced, this could improve the health and quality of life for many community-dwelling older adults.

\section{The Social Ecological Model}

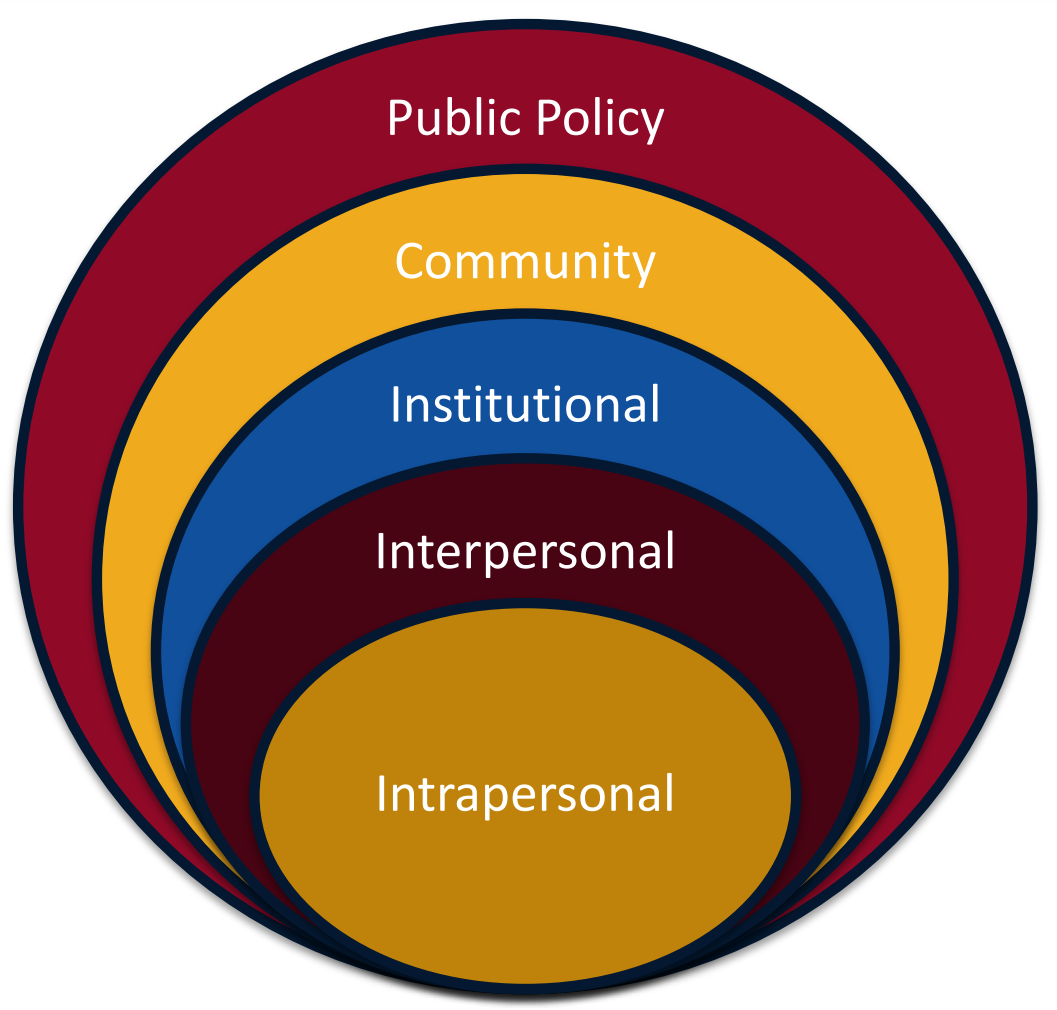

Factors Influencing Nutritional Risk

Intrapersonal

- Knowledge

- Beliefs

- Health status

- Skills

- Socio-economic status

- Physiological changes that occur with aging

\section{Interpersonal}

- Low social support

- Social isolation

- Eating alone

- Changes in interpersonal relationships

- Living Alone

\section{Institutional}

- Health care organizations

- Screening programs

- Dining environment

- Social norms

\section{Community}

- Community resources

- Transportation

- Walkability of neighbourhoods

- Proximity, number and design of grocery stores

- Number and design of other food outlets

Public Policy

- Screening for Nutritional Risk

- Program availability and accessibility

- Poverty and Food insecurity

- Food costs and availability

- Health care

The poster is based on a paper written for a course entitled Critical Analysis of Aging Theories. The author would like to thank Kevin Woo, RN, BScN, MSc, PhD, the instructor for that course, for his comments on the paper. To read the paper which includes all the references for this poster, please access the QR code.

For further information contact christine.mills@queensu.ca Twitter: @ChrisMIIsRD 